\title{
A mathematical model of Noggin and BMP densities in adult neural stem cells
}

\author{
Kamila Larripa ${ }^{\mathrm{a}}$ and Angela Gallegos ${ }^{\mathrm{b}}$ \\ ${ }^{a}$ Department of Mathematics, Humboldt State University, Arcata, CA, USA; ${ }^{b}$ Department of Mathematics, \\ Loyola Maramount University, Los Angeles, CA, USA
}

\begin{abstract}
New neurons are generated in the adult hippocampus throughout life by neural stem cells (NSCs) in a dynamic process responsive to external signalling cues. NSCs in the adult hippocampus divide infrequently, and it has been shown that bone morphogenetic protein (BMP) modulates their quiescence. Infusion of Noggin, a BMP antagonist, blocks this signalling. We investigate the balance of BMP and Noggin in this particular niche and qualitatively reproduce experimental results obtained and qualitatively reproduce experimental results with a one-dimensional reaction-diffusion model. We use the model to connect BMP signalling profiles with specific cellular outcomes and to determine whether the transient infusion of BMP leads to a signalling profile which can be reversed by the infusion of Noggin. Additionally, we analyse the role of diffusion in this system for generating signalling profiles with dramatically different cell-fate outcomes and show that diffusion-driven instability is not possible in our system of reactiondiffusion equations.
\end{abstract}

Abbreviations: NSC: Neural Stem Cell; SVZ: subventricular zone of the hippocampus; SGZ: subgranular zone of the hippocampus; BMP: bone morphogenetic protein

\section{ARTICLE HISTORY}

Received 31 March 2017

Accepted 23 October 2017

\section{KEYWORDS}

Adult neural stem cells; adult hippocampus; quiescence; partial differential equations; mathematical biology

\section{Introduction}

\subsection{Stem cells and quiescence}

Stem cells are how we all begin: undifferentiated cells that go on to develop into any of the more than two hundred types of cells present in the adult human body. They are characterized by the ability to renew themselves through mitotic cell division or to differentiate into specialized cell types.

Many stem cells are quiescent, meaning they are not actively dividing to create new cells, but can re-enter the cell division cycle and proliferate at some future time in response to appropriate signals. For example, lymphocytes might come out of quiescence to mount an immune response. The ability to enter and exit quiescence appropriately and to remain

CONTACT Kamila Larripa kamila.larripa@humboldt.edu

(c) 2017 The Author(s). Published by Informa UK Limited, trading as Taylor \& Francis Group.

This is an Open Access article distributed under the terms of the Creative Commons Attribution License (http://creativecommons.org/ licenses/by/4.0/), which permits unrestricted use, distribution, and reproduction in any medium, provided the original work is properly cited. 
viable while quiescent is central to an organism's ability to maintain homeostasis. In mammals, cells enter quiescence in response to an absence of growth factors or situational cues (Coller, 2011) from their local environment, or niche.

The term niche is broad and encompassing. The detailed mechanisms with specific niches are extremely varied. For example, a niche may be composed of cells, or of cells along with extracellular structures, and may include secreted factors or cell surface components working in tandem to control cell renewal, maintenance and survival (Lander et al., 2012). It is hypothesized that niches are agents of feedback control (Lander et al., 2012). Stem cell pools aren't maintained at a constant size, but fluctuate in population, even under homeostatic conditions. Therefore, the niche is more than just a supportive environment for stem cell functioning. It is a device for communicating the needs of a tissue back to the stem cells that maintain it. Broadly understanding how niches receive and transmit this information is an important question, but because niches are so varied, this question must be addressed on a niche-by-niche basis.

Quiescence of stem cells is critical to ensure lifelong tissue maintenance and to protect the stem cell pool from premature exhaustion. The long-term maintenance of stem cells is a function of their interaction with their specific niche. The interaction regulates stem cell-specific properties, including self-renewal, multi-potentiality and quiescence in the cell cycle. Therefore, identifying the mechanism that regulates the quiescent state of adult Neural stem cell (NSC) is necessary to understanding how these populations are maintained (Kawaguchi, Furutachi, Kawai, Hozumi, \& Gotoh, 2013; Mira et al., 2010).

\subsection{Neural stem cells}

NSCs are the self-renewing, multipotent cells that generate the main phenotypes of the nervous system. They are important during development, giving rise to the diversity of neurons, astrocytes and oligadendrocytes in the developing Central Nervous System. They play an important role in adult learning and hippocampal plasticity, and, perhaps most importantly, disease and prevention. Their response during stroke, multiple sclerosis, cancer, and Parkinson's disease is an area of active research. For example, it has been shown that NSCs migrate to brain tumours (Snyder, 2011) or respond to strokes. A fundamental question is how pool size of the population of NSCs is maintained over long periods of time while continuously producing neurons (Kawaguchi et al., 2013).

In the adult brain, NSCs are maintained in the subventricular zone (SVZ) and the subgranular zone (SGZ) of the hippocampus. In these regions, the NSCs divide infrequently, and are characterized as quiescent. Bone morphogenetic protein (BMP) signalling affects adult NSC proliferation and cellular differentiation (Bonaguidi et al., 2008), and has recently been shown to play a role in regulating quiescence (Johnston \& Lim, 2010).

\subsection{BMP and Noggin}

Research by Bonaguidi et al. (2008) has shown that BMP can affect NSC proliferation and cellular differentiation. In the SGZ of the adult hippocampus, high levels of BMP are found with quiescent NSCs. When BMP was blocked with Noggin (a signalling protein which inhibits BMP), there was evidence of cell self-renewal, proliferation and multipotentiality. In fact, NSC maintenance requires continual Noggin exposure, which implicates BMP as 
a signal of NSC aging. It was concluded that the hippocampus contains a population of NSCs which can be expanded by blocking BMP (Bonaguidi et al., 2008).

More recently, Mira et al. (2010) has shown that BMP signalling also affects the quiescent SGZ NSCs. The balance between active, proliferating cells and a quiescent pool has important implications for addressing injury or disease. Indeed, by maintaining reservoirs of stem cells that can re-enter the cell cycle, an organism can respond to different physiological demands as they arise. It has been demonstrated that BMP signalling regulates the quiescence of these NSCs, preventing their proliferation and maintaining their stores (Mira et al., 2010).

The binding of BMP on the cell surface is part of a canonical signalling pathway. Roughly, once BMP binds to a receptor on the cell surface, the receptor rotates, leading to phosphorylation and this in turn begins signalling within the cell. This process keeps cells from proliferating. When BMP is sequestered by its antagonist Noggin to form a complex, it is unavailable to bind to receptors on the cell surface. This interplay between BMP and Noggin is crucial to the signalling profile and to the future fate of stem cells.

Although there is vast literature on BMP and Noggin signalling (Lim et al., 2000; Nerurkar, Mahadevan, \& Tabin, 2017; Stantzou et al., 2017), the work that specifically concerns quiescence of NSCs in the adult hippocampus SGZ through infusion of Noggin is more limited, and there is disparity regarding the precise role of BMP signalling in the SGZ in these reports. For example, Mira et al. (2010) suggests that long-term Noggin treatment depletes the stem cell pool, implying that NSCs in the SGZ have a limited ability to self-renew. However, Bonaguidi et al. (2008) have found the effects of Noggin are renewable. Although the details remain to be elucidated, it is agreed that the balance between Noggin and BMP is critical to the regulation of adult hippocampal neurogenesis. For our work below, we will rely specifically on work that has infused Noggin directly into the hippocampus or has added Noggin to hippocampal cultures. We then attempt to connect the BMP signalling profiles generated by our model to the experimental outcomes observed (Figure 1).

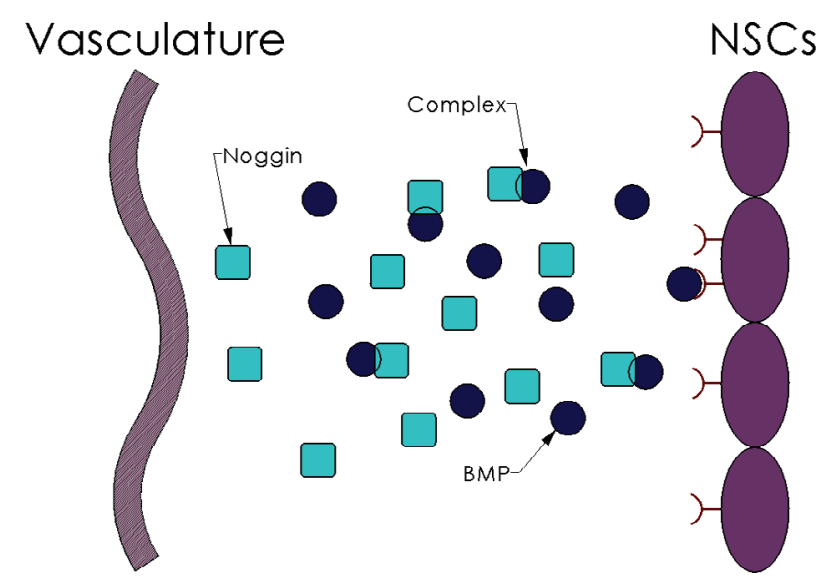

Figure 1. Noggin is released from the vasculature and can bind with BMP forming a complex.

Notes: This binding prevents BMP from binding to the BMP receptors on the NSCs. Without BMP signalling, NSCs emerge from quiescence and actively divide. 
Extracellular BMP-binding proteins can cause remarkably context-specific gains or losses in signalling. In most cases, extracellular Noggin, and in general BMP-binding proteins, act as inhibitors by binding to BMP, keeping them away from receptors on the cell surface. However, when BMP is bound to a protein such as Noggin or Chordin, the complex is able to diffuse much faster through the extracellular space (Ben-Zvi, Shilo, Fainsod, \& Barkai, 2008). The binding protects it from receptor-mediated endocytosis and turnover (O'Connor, Umulis, Othmer, \& Blair, 2006) allowing the complex to diffuse more rapidly. It has also been shown (Umulis et al., 2009) that very tight binding is required between the the activator and the inhibitor for this shuttling mechanism to be effective. In similar niches, an extracellular protease cleaves the BMP binding protein to release the ligand (Umulis et al., 2009). This returns BMP to the pool, and eliminates the inhibitor. It is suggested that that this cleavage has provided a paradigm for understanding signalling regulation in adult tissues and that this model may apply to other BMP/TGF $\beta$ signalling relationships (De Robertis et al., 2001) so we make this assumption in our model also.

Mira et al. (2010) hypothesize that there exists a double gradient of the BMP signalling profile along the neurogenic pathway, and that Noggin signalling counteracts BMP signalling, leading to stem cell proliferation. It has been shown that the establishment of a BMP gradient in some cases is really a result of long-range inhibition by Noggin and Chordin (Jones \& Smith, 1998). This work was done in the Xenopus embryo, but the conservation of function across species and locations suggests something similar may be at work in the adult hippocampus. BMP is prevalent, so neurogenesis might be restricted to regions expressing BMP antagonists such as Noggin (Lim et al., 2000).

We specifically explore the interactions between Noggin, BMP and Noggin-BMP complexes within the niche of the adult hippocampus with a reaction-diffusion model. This focus is based on relevant experiments found in Mira et al. (2010) and Bonaguidi et al. (2008). We use the model to predict the signalling profile generated by BMP and Noggin under a variety of biologically relevant regimes. The model also allows us to investigate effects of Noggin or BMP infusion through boundary conditions. We qualitatively reproduce experiments performed by Mira et al. (2010), and use our model and simulation to explore whether the transient infusion of BMP could reversibly increases quiescence of SGZ NSCs. (This question was posed in Johnston and Lim, 2010 when summarizing Mira et al., 2010.) Our model connects the BMP signalling profile to outcomes obtained experimentally. We show that the transient infusion of BMP leads to a signalling profile known to induce quiescence and that this signalling profile can be significantly altered by the infusion of Noggin.

We discuss the model in the next section and address 'in silico' experiments related to the work in Mira et al. (2010) below.

\section{Mathematical model}

To further elucidate the roles of Noggin and BMP in this specific niche, we write and analyse a mathematical model on a simplified geometry. Continuum models are prevalent in mathematical modelling of biology, and their use is justified when the length scale of the problem of interest is larger than the length scale of the underlying microscopic elements of the model (Spill et al., 2015). We justify the use of a continuum model for several reasons. First, experiments were conducted by Mira et al. (2010) that tested whether cell 


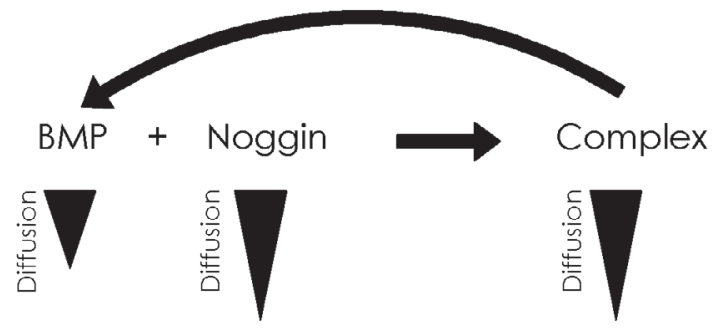

Figure 2. BMP, Noggin and the complex all diffuse, but Noggin diffuses more slowly.

Note: BMP and Noggin can combine to form the complex, and the complex can be cleaved, returning BMP to the pool.

density influenced BMP function and no significant difference was found between sparse and densely packed adult hippocampal NSCs. Second, the length scale of the BMP ligand is significantly smaller than that of the intercellular space. Third, averaged densities of Noggin, BMP and the Noggin-BMP complex form well defined, continuous functions on the macroscale (Spill et al., 2015), and the large cell population offers further justification for the use of a continuum model (Percus \& Childress, 2015) (Figure 2).

We model our system as a one-dimensional strip representing the space just outside of the NSCs. We have written a system of three reaction-diffusion-degradation equations for the density of Noggin $(N)$, BMP $(B)$, and the Noggin-BMP complex $(C)$ found in this region.

The equations are as follows:

$$
\begin{aligned}
\frac{\partial N}{\partial t} & =D_{N} \frac{\partial^{2} N}{\partial x^{2}}-k_{\mathrm{BMP}} N B-\lambda_{1} N \\
\frac{\partial B}{\partial t} & =D_{B} \frac{\partial^{2} B}{\partial x^{2}}-k_{\mathrm{BMP}} N B+\lambda_{2} C \\
\frac{\partial C}{\partial t} & =D_{C} \frac{\partial^{2} C}{\partial x^{2}}+k_{\mathrm{BMP}} N B-\lambda_{2} C
\end{aligned}
$$

The first equation states that the density of Noggin changes in time due to diffusion, depletion due to combining with BMP to form a complex, or degradation. The second equation tracks the density of BMP, which is affected by diffusion, reaction and also degradation of the complex, which increases the amount of free BMP. The third equation states that the density of the complex changes in time due to diffusion and from the binding of Noggin and BMP.

Here, degradation eliminates the Noggin in the complex, freeing BMP. We make the assumption that the turnover of the complex frees BMP because this is the case for the BMP-Chordin complex in the Xenopus embryo (in this case, BMP is cleaved by the Xolloid metalloprotease and then reactivated (Piccolo et al., 1997)), and, in parallel studies in Drosophila, this occurs when the DPP (analagous to BMP) antagonist Sog is cleaved by Tolloid (Marqués et al., 1997; Oelgeschläger, Larraín, Geissert, \& De Robertis, 2000). It is suggested that Chordin has provided a paradigm for understanding signalling regulation in adult tissues and that this model may apply to other BMP/TGF $\beta$ signalling relationships (De Robertis et al., 2001) so we make this assumption in our model.

We also assume that the degradation of free BMP is negligible compared to that of free Noggin and to the complex. We make this assumption under the paradigm described 
above, in which a currently unknown protease cleaves the complex and also affects free Noggin, but does not impact BMP. Similar mathematical assumptions have been made in Eldar et al. (2002). This assumption is also supported by estimates of the half life of of BMP (approximately an hour) due to internalization and natural degradation (Inomata, Shibata, Haraguchi, \& Sasai, 2013). Internalization is not a factor in our geometry except at the right boundary condition, and we capture internalization there rather than in the reaction-diffusion equation.

Degradation rates for free and bound Noggin differ. Chordin/Sog degradation by a protease is dependent on it being bound to the ligand (Ben-Zvi et al., 2008; Eldar et al., 2002; Mizutani et al., 2005). In other words, an antagonist (for our model, Noggin) that is not bound to a BMP is a relatively ineffective substrate for the protease. This has been been demonstrated in vitro for Drosophila proteins (Marqués et al., 1997), however this mechanism is not explicitly known for Noggin and BMP and we recognize this assumption should be further validated with biological experiments.

We assume that initially there is a uniform density of BMP throughout the intercellular space, while Noggin is absent (it will be infused to the intercellular space through the surrounding vasculature or through a pump during experiments). The complex is also absent, as BMP hasn't yet encountered Noggin. These assumptions can be stated mathematically as the following initial conditions:

$$
\begin{aligned}
N(x, 0) & =0 \\
B(x, 0) & =B_{\text {unif }} \\
C(x, 0) & =0
\end{aligned}
$$

We assume without loss of generality that the vasculature is on the left boundary $(x=0)$ and the cell surface is on the right boundary $(x=L)$, where the length of the space we are considering is $L$.

On the left, we assume that Noggin enters with a constant flux. The flux of BMP and of the BMP-Noggin complex is zero from the vasculature. On the right, we assume that Noggin and the Noggin-BMP complex have zero flux, and that a flux of BMP represents the binding of this ligand to receptors on the cell surface. We let this flux be dependent on the amount of BMP present at the cell surface. There is little data regarding these in vivo values and we discuss our estimates and justification below in Section 2.2.

The boundary conditions are as follows:

$$
\begin{aligned}
& \frac{\partial N}{\partial x}(0, t)=f \\
& \frac{\partial B}{\partial x}(0, t)=0 \\
& \frac{\partial C}{\partial x}(0, t)=0 \\
& \frac{\partial N}{\partial x}(L, t)=0 \\
& \frac{\partial B}{\partial x}(L, t)=m \frac{B(L, t)}{B(L, t)+1}
\end{aligned}
$$




$$
\frac{\partial C}{\partial x}(L, t)=0
$$

We assume that diffusion of the free ligand (BMP) is slow relative to the Noggin-BMP complex as well as compared to Noggin. Mathematically, this means $D_{B}<<D_{C}$ and $D_{B}<<D_{N}$. These assumptions are well supported by the literature (Ben-Zvi et al., 2008; Zaraisky, 2007) and further supported by the relative size of these proteins.

\subsection{Non-dimensionalization}

Non-dimensionalizations are not unique, so we choose one that highlights the features of our model on which we wish to focus, minimizes the number of parameters and keeps the range of the non-dimensional parameters small for the numerical work below. Our scales are $\ell=\sqrt{D_{C} / \lambda_{2}}, \tau=\frac{1}{\lambda_{2}}, \theta=B_{\text {unif }}$, the initial concentration of BMP in the intercellular space.

This yields the following groups of non-dimensional parameters $\alpha=\frac{k_{\mathrm{BMP}} \theta}{\lambda_{2}}, \beta=\frac{\lambda_{1}}{\lambda_{2}}$, $D_{N C}=\frac{D_{N}}{D_{C}}$ and $D_{B C}=\frac{D_{B}}{D_{C}}$. Our non-dimensional equations become the following (here and below, $N, B$, and $C$ refer to non-dimensional variables):

$$
\begin{aligned}
\frac{\partial N}{\partial t} & =D_{N C} \frac{\partial^{2} N}{\partial x^{2}}-\alpha N B-\beta N \\
\frac{\partial B}{\partial t} & =D_{B C} \frac{\partial^{2} B}{\partial x^{2}}-\alpha N B+C \\
\frac{\partial C}{\partial t} & =\frac{\partial^{2} C}{\partial x^{2}}+\alpha N B-C
\end{aligned}
$$

The four dimensionless parameters relate diffusion rates or reaction rates and are summarized in Table 1, and dependent on the values of the dimensional parameters in Table 2.

Similarly, we non-dimensionalize the initial conditions (here $x$ represents scaled space):

$$
\begin{aligned}
N(x, 0) & =0 \\
B(x, 0) & =1 \\
C(x, 0) & =0
\end{aligned}
$$

The non-dimensional boundary conditions (where $x$ represents scaled space and $t$ represents scaled time) are

$$
\begin{aligned}
& \frac{\partial N}{\partial x}(0, t)=f \\
& \frac{\partial B}{\partial x}(0, t)=0 \\
& \frac{\partial C}{\partial x}(0, t)=0 \\
& \frac{\partial N}{\partial x}(1, t)=0 \\
& \frac{\partial B}{\partial x}(1, t)=m \frac{B(1, t)}{B(1, t)+1}
\end{aligned}
$$


Table 1. Non-dimensional parameter meanings and values.

\begin{tabular}{|c|c|c|c|c|}
\hline $\begin{array}{l}\text { Nondimensional } \\
\text { parameter }\end{array}$ & Definition & Biological meaning & $\begin{array}{l}\text { Possible } \\
\text { range }\end{array}$ & $\begin{array}{l}\text { Value for numerical } \\
\text { experiments }\end{array}$ \\
\hline$\alpha$ & $\frac{k_{\mathrm{BMP}} B_{\mathrm{unif}}}{\lambda_{2}}$ & $\begin{array}{l}\text { Binding rate of Noggin and BMP compared to } \\
\text { degradation rate of free Noggin }\end{array}$ & $.2-2$ & 1 \\
\hline$\beta$ & $\frac{\lambda_{1}}{\lambda_{2}}$ & $\begin{array}{l}\text { Ratio of degradation rates for free and bound } \\
\text { Noggin }\end{array}$ & .12 & .12 \\
\hline$D_{N C}$ & $\frac{D_{N}}{D_{C}}$ & $\begin{array}{l}\text { Ratio of diffusion coefficients for Noggin and } \\
\text { the complex }\end{array}$ & $1.14-11.4$ & 1.14 \\
\hline$D_{B C}$ & $\frac{D_{B}}{D_{C}}$ & $\begin{array}{l}\text { Ratio of diffusion coefficients for BMP and the } \\
\text { complex }\end{array}$ & $.01-.1$ & .1 \\
\hline$f$ & $\frac{\partial N}{\partial x}(0, t)$ & $\begin{array}{l}\text { Non-dimensional Neumann boundary condi- } \\
\text { tion for Noggin }\end{array}$ & -7.9 to -.008 & -.008 \\
\hline$m$ & $\frac{\partial B}{\partial x}(1, t)$ & $\begin{array}{l}\text { Non-dimensional Neumann boundary condi- } \\
\text { tion for BMP }\end{array}$ & -.005 to -.00005 & -.00005 \\
\hline$k$ & & $\begin{array}{l}\text { Nondimensional scaling constant for Hill } \\
\text { function in autorcrine signalling }\end{array}$ & Unknown & .0000065 \\
\hline
\end{tabular}

$$
\frac{\partial C}{\partial x}(1, t)=0
$$

where $f$ and $m$ are now appropriately scaled.

\subsection{Parameter choices}

We note that many of the results are dependent on the values chosen for the different parameters, and specifically on their relationship to each other in the non-dimensionalized equations. It is generally agreed that Noggin diffuses roughly one to two orders of magnitude faster than BMP (Zaraisky, 2007). Because we are considering the intercellular space, to determine the diffusion coefficient, it is necessary to consider the presence of the extracellular matrix. By diffusing through extracellular matrices more efficiently than members of the TGF- $\beta$ superfamily, Noggin may have a principle role in creating morphogenic gradients.

There is little data regarding the in vivo rate constants and diffusion coefficients, however range estimates are listed in Table 2. It is also suggested that Noggin can alter BMP's effective diffusion coefficient (Balemans \& Van Hul, 2002), so parameters are contextspecific.

In Ben-Zvi et al. (2008) many parameters analogous to our system range over three orders of magnitude. We list the ranges of these in Table 2 below for diffusion coefficients and reaction rates unless we a primary measurement is available.

Cell gaps in the hippocampus can be as small as .005 $\mu \mathrm{m}$ (Syková \& Nicholson, 2008). However, we are specifically considering the region between the vasculature which secretes Noggin and the NSCs. Hama et al. (2011) estimate the distribution of distance of nuclei of NSCs from the vasculature with fluorescence imaging (see Figure 5(g)). We take an average of $25 \mu \mathrm{m}$ to determine our length scale. Degradations rates for bound Noggin are listed in Ben-Zvi et al. (2008) and Umulis et al. (2006).

The diffusion coefficient for Noggin was measured to be $11.4 \frac{\mu \mathrm{m}^{2}}{\mu \mathrm{s}}$ using fluorescence recovery after photobleaching (FRAP) Eroshkin et al. (2016). The diffusion coefficient for 
BMP is often estimated using the diffusion coefficient for DPP in the Drosophila wing disc. An estimate for the DPP diffusion coefficient in Drosophila wing discs is $.10 \frac{\mu \mathrm{m}^{2}}{\mathrm{~s}}$ (Yu et al., 2009). However, in the zebrafish embryo, it's two orders of magnitude larger (Yu et al., 2009), so applying experimentally derived values to a novel biologically context must be done so carefully.

We assume the diffusion of BMP is accelerated when bound to Noggin, so the complex diffuses faster than BMP. A similar assumption was made regarding Chordin and BMP in Ben-Zvi et al. (2008). This assumption has been questioned in contexts where diffusion is fairly rapid (for example the Xenopus embryo) (Francois, Vonica, Brivanlou, \& Siggia, 2009). However, due to the tortuosity of the brain, the adult hippocampus is a context where this is a valid assumption.

Diffusion in the brain is constrained by the volume fraction and tortuosity. Measurements with real-time iontophoresis (RTI) methods and theoretical modelling suggest parameter rates that would be applicable in the adult hippocampus (Syková \& Nicholson, 2008), and we use these when running our model. Empirically it has been found that diffusion of molecules is roughly 3-5 fold times slower in the brain than in water, and more specifically, in certain niches, the diffusion between tissue microvasculature and cells depends intricately on total tissue volume (Syková \& Nicholson, 2008).

For the degradation rate of Noggin, we estimate it by using the fact that Noggin is more stable than Chordin (Inomata et al., 2013) and that measurements for Chordin's degradation rate exist and are estimated to be $.001 \frac{1}{\mathrm{~s}}$. Chordin was found to have a halflife of thirty minutes, and Noggin was stable for six hours after injection into an embryo (Inomata et al., 2013), so we scale Chordin's degradation rate by 12 to estimate free Noggin's degradation rate to be $012 \frac{1}{\mathrm{~s}}$. This is within the range of the free antagonist degradation rate suggested in the mathematical model presented by Ben-Zvi et al. (2008) (estimates were $10^{-3}$ to $10^{-1} \frac{1}{\mathrm{~s}}$ ). We also assume that Noggin enjoys some protection from degradation when bound to BMP. We make this assumption because in the Chordin-paradigm, it has been shown that an unbound antagonist was a relatively ineffective substrate for the protease leading to cleavage and degradation (Marqués et al., 1997) We choose a range for the slower degradation that is one to two orders of magnitude smaller, and agrees with the range selected by Ben-Zvi et al. (2008) for an analogous inhibitor.

The degradation rate of BMP, was meausured to be $.0002 \frac{1}{\mathrm{~s}}$ (Inomata et al., 2013). Because this rate is an order of magnitude smaller than that for Noggin, and because we are capturing the internalization of BMP through the right boundary condition, we omit a degradation term for BMP from our model equations.

The binding rate of Chordin to immobilized BMP2 has been measured to be $2.8 \times$ $10^{5} \mathrm{M}^{-1} \mathrm{~s}^{-1}$ by Rentzsch et al. (2006). However, the authors indicated that the binding values for BMP binding proteins determined via the Biacore analyses implemented can differ by one order of magnitude, depending on which of the tested partners is immobilized. An additional problem with this estimate is that it is in terms of a three-dimensional concentration. Because our model is defined on a simplified one-dimensional geometry, there are not references in the experimental literature that are exactly applicable. For this reason, using an estimate from a previous one-dimensional mathematical model Ben-Zvi et al. (2008) is justified.

For the boundary conditions, we estimate the constant flux of Noggin on the left by recognizing that Noggin is functionally and structurally similar to Chordin and using values 
Table 2. Model parameters.

\begin{tabular}{llccc}
\hline Parameter & \multicolumn{1}{c}{ Meaning } & Dimensions & Value & Reference \\
\hline$D_{N}$ & Diffusion of Noggin & $\frac{\text { length }}{\text { time }^{2}}$ & $11.4 \frac{\mu \mathrm{m}^{2}}{\mathrm{~s}}$ & Eroshkin et al. (2016) \\
$D_{B}$ & Diffusion of BMP (note this is slower) & $\frac{\text { length }}{\mathrm{time}}$ & $.1 \frac{\mu \mathrm{m}^{2}}{\mathrm{~s}}$ & Yu et al. (2009) \\
$D_{C}$ & Diffusion of complex & $\frac{\text { length }}{\mathrm{time}}$ & $1-10 \frac{\mu \mathrm{m}^{2}}{\mathrm{~s}}$ & Ben-Zvi et al. (2008) \\
$k_{\mathrm{BMP}}$ & Binding rate of Noggin and BMP & $\frac{1}{\text { concentration)(time) }}$ & $.012 \frac{1}{\mu \mathrm{ms}}$ & Inomata et al. (2013) \\
$\lambda_{1}$ & Degradation rate of free Noggin (note this is slower) & $\frac{1}{\text { time }}$ & $10^{-3}$ to $10^{-1} \frac{1}{\mathrm{~s}}$ Ben-Zvi et al. (2008) \\
$\lambda_{2}$ & Degradation rate of bound Noggin & $\frac{1}{\text { time }}$ & $10^{-1} \frac{1}{\mathrm{~s}}$ & Ben-Zvi et al. (2008) \\
$L$ & Length of intercellular space & length & $25 \mu \mathrm{m}$ & Hama et al. (2011) \\
$B_{\text {unif }}$ & Initial concentration of BMP & concentration & $.2 \mu \mathrm{M}$ & Ben-Zvi et al. (2008) \\
$f$ & Flux of Noggin entering from vasculature & $\frac{\text { concentration }}{\text { length }}$ & $10-10^{3} \frac{\mu \mathrm{M} \mu \mathrm{m}}{\mathrm{s}}$ & Ben-Zvi et al. (2008) \\
$m$ & Flux of BMP due to binding with cell receptors & $\frac{\text { concentration }}{\text { length }}$ & $.1 \frac{\mu \mathrm{M} \mu \mathrm{m}}{\mathrm{s}}$ & Karim et al. (2012) \\
\hline
\end{tabular}

from Ben-Zvi et al. (2008). The pump features used in some of the experiments discussed below could alter the flux on the left, so we consider a range of values. For the constant flux of BMP on the right, we use estimates from Karim, Buzzard, and Umulis (2012). In both cases, we solve for the derivative in the flux equation. For example, $J_{B}=-D_{B} \frac{\partial B}{\partial x}$, so $\frac{\partial B}{\partial x}$ is in the range of $[-1,-.01]$ with units of concentration per length. We then scale these values according to our non-dimensionalization above, meaning we multiply by the length scale and divide by the concentration scale. This gives a range for non-dimensional $m$ of $[-.005,-.00005]$ for the non-dimensional boundary condition $\frac{\partial B}{\partial x}(1, t)=m \frac{B(1, t)}{B(1, t)+1}$.

Because experimental values of parameters listed above come from in vitro experiments or in vivo experiments in a different model organism, they cannot be relied upon without caution. Moreover, FRAP kinetics may fail to provide information about morphogen transport when transport is relatively fast and degradation is relatively slow (Zhou et al., 2012). The challenges with experimentally measuring parameters or applying measured values to a new context (such as applying measured parameters for diffusion in the Xenopus embryo to diffusion of the adult human hippocampus) can be successfully mitigated through mathematical modeling. For example, Kicheva et al. (2007) fit analytical solutions to reaction diffusion degradation equations to FRAP recovery curves to estimate diffusion coefficients and reaction rates, and Ben-Zvi et al. (2008) use mathematical modelling to find parameter ranges which give biologically valid solutions, in which the embryo is scaled appropriately. Some of our parameter values come from previous mathematical modelling work for this reason.

Additionally, there are very few measurements of rates for Noggin in particular, either alone or in a complex with BMP, so we must rely on estimates from previous mathematical modelling work and we perform a sensitivity analysis below. Ideally, future lab experiments will provide estimates for these values and validate the ranges found from modelling.

\section{Numerical results and analysis}

All of the numerical results presented in this section and subsections use the non-dimensional parameter values given in Table 1. Individual parameter exceptions are 


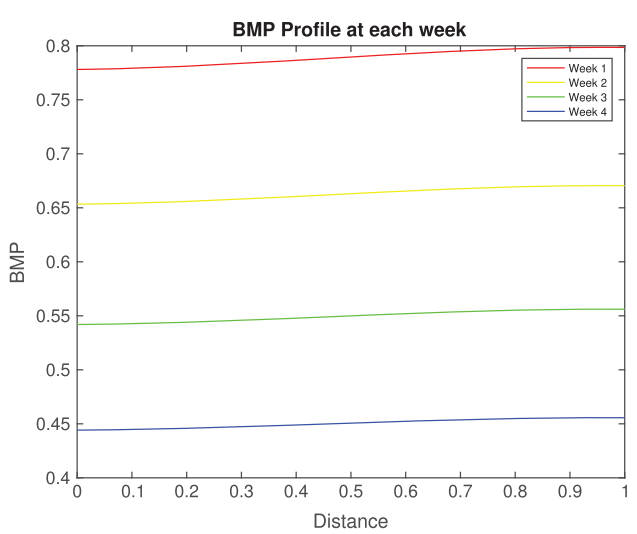

(a) BMP concentration levels in the intercellular space at weekly intervals. The signaling level is the BMP concentration at the right boundary.

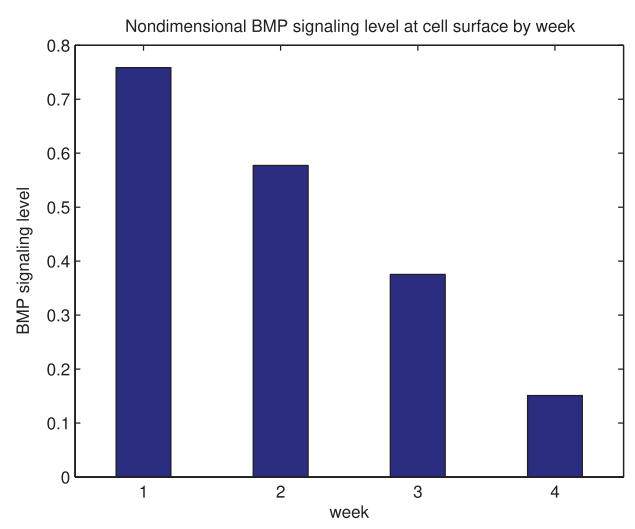

(b) The BMP signaling level at the cell surface by week. The falling levels due to Noggin infusion cause stem cells to emerge from quiescence.

Figure 3. Results from the base model.

Note: The influx of Noggin reduces the BMP signalling level and leads to NSCs emerging from quiescence.

highlighted in their respective subsections when modifications represent the experiments. We solved our equations under a variety of biologically relevant boundary conditions (discussed below) using Matlab's build in solver pdepe. We discuss specific results below.

\subsection{Base model}

We numerically solve the non-dimensional model presented in Section 2.

This model represents the intercellular space, with the vasculature on the left, and the NSCs on the right. We assume a constant initial level of BMP, and begin secreting Noggin from the vasculature on the left and allow BMP to bind to cell receptors on the right. We consider the signalling profile of BMP at specific time periods that coincide with experiments discussed below.

We find that the influx of Noggin leads to a monotonically decreasing signalling profile of BMP at the cells surface, as illustrated in Figure 3.

\subsection{Autocrine or paracrine signalling}

Positive feedback occurs when a signal induces the production of itself, and such loops can prolong signalling (Freeman, 2000). It is suggested that adult hippocampal neural stem cells secrete BMP, which may regulate proliferation in an autrocrine fashion (Mira et al., 2010). It is also known that precursors secrete BMP, affecting signalling in an autocrine/paracrine manner (Bonaguidi et al., 2008). We modify our model above to include production of BMP from within the cells as a function of the amount of BMP present. This feedbackmediated induction of BMP is represented mathematically by adding a production term to the equation governing the evolution of BMP that is an increasing Hill function of the present BMP levels (Badugu, Kraemer, Germann, Menshykau, \& Iber, 2013; Ben-Zvi et al., 2008) (Figure 4). 


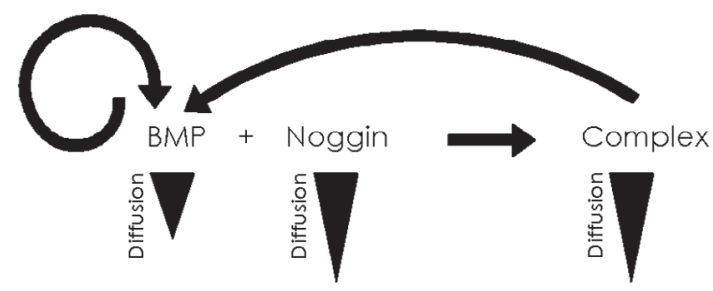

Figure 4. The model incorporating autocrine or paracrine signalling.

We achieve this by adding the term $k \frac{B^{4}}{\left(B+T^{4}\right)}$ to the non-dimensional Equation (14). This function is a Hill function starting at 0 and rising to $k$, and represents the positive induction of BMP signalling by itself.

$$
\frac{\partial B}{\partial t}=D_{B C} \frac{\partial^{2} B}{\partial x^{2}}-\alpha N B+C+k \frac{B^{4}}{\left(B^{4}+T^{4}\right)}
$$

Our results indicate that BMP signalling continues to grow each week. It is unlikely that this scenario would allow stem cells to emerge from quiescence unless there is another mechanism at play or unless cells attenuate to BMP signalling levels.

\subsection{Infusion of Noggin experiment}

Mira et al. (2010) conducted experiments investigating the effects of transient Noggin infusion on SGZ NSC proliferation. After infusing Noggin for 1 week into the adult mouse brain ventricle, they found neuronal production increased two-fold. This effect was transient and after another three weeks there were fewer label retaining cells, suggesting that quiescent NSCs had been partially depleted. Bonaguidi et al. (2008) showed that continuous Noggin release increased the number of dividing cells. The difference in results is thought to be partially attributed to using a pump versus continuous release. Our model captures the continuous release mechanism.

To reflect the continuous release of Noggin, we alter the boundary conditions in the following way. We first allow the left boundary condition to have a constant flux of Noggin for 1 week. We then shut that flux off and look at the solution at weekly intervals to compare with the data in Mira et al. (2010). We allow BMP to flux out at the right boundary, as before.

We compare our results with the results in (Mira et al., 2010), Supplemental Material Figure 6 and with the trend explained in Bonaguidi et al. (2008). With continuous Noggin exposure, the BMP signalling level reduces each week, meaning cells are coming out of quiescence. It is interesting that the BMP signalling level continues to decrease even after the Noggin infusion ceases. This suggests that even a transient influx of Noggin is enough to bring stem cells out of quiescence (Figure 5).

\subsection{Infusion of BMP theoretical experiment}

The summary paper by Johnston and Lim (2010) posed the following question in relation to the first set of Mira's experiments: Does the transient infusion of BMP reversibly increase the quiescence of SGZ NSCs? We use our model to make a prediction. 


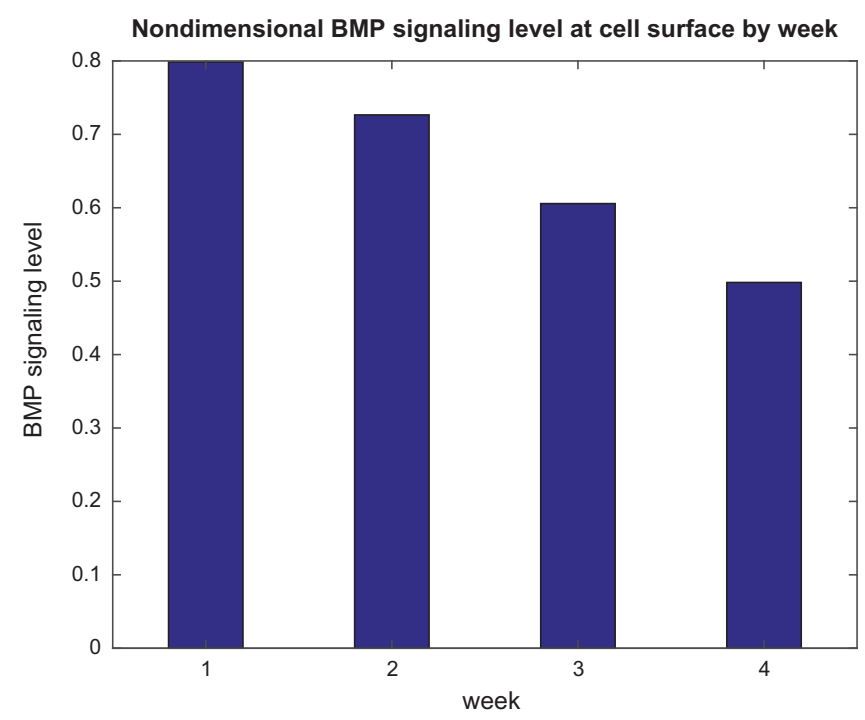

Figure 5. BMP signalling level at the cell surface at weekly intervals after Noggin has been infused for one week.

Note: The infusion of Noggin results in lower BMP levels and cells emerging from quiescence.

Experiments focusing on the Zebrafish (Chapouton et al., 2010) have shown that activated NSCs can be reversed to the quiescent state by signalling. However, in this particular case, the signalling molecule was Notch, not BMP. This leads us to investigate whether something similar occurs in adult NSCs in the adult hippocampus with BMP and Noggin dynamics.

We consider our base model, but alter the boundary conditions in the following way. We first allow the left boundary condition to have a constant flux of BMP. We continue this for 1 week. We then shut that off and flux in Noggin to see if we are able to reverse the signalling profile so that it matches the profile that corresponds to NSCs emerging from quiescence. We allow BMP to flux out on the right at a rate proportional to the amount of BMP present. Our model does not give us a quantitative value of the number of radial SGZs, but by considering the signalling profile and considering if we can return to the original profile, we can suggest that the process is reversible. This situation is analogous to the base model with Noggin fluxing in from the vasculature. The initial administration of BMP increases its concentration, but Noggin can reverse this effect. Thus, effects of BMP can be reversed by administering Noggin (Figure 6).

\section{Mathematical analysis}

\subsection{Sensitivity analysis}

Sensitivity analysis (SA) is a method to quantitatively determine how a model's behavior depends on model parameterization (Ingalls, 2008). The sensitivity of any species concentration with respect to a given parameter is defined as the partial derivative of the species with respect to the parameter. The sensitivity coefficient (SC) determines how the species 


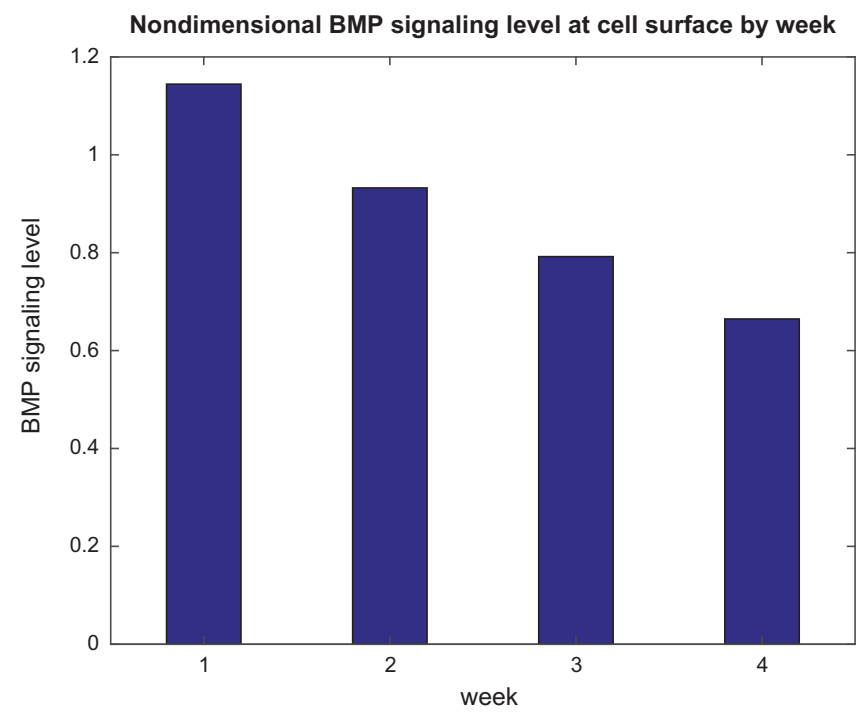

Figure 6. BMP signalling level at the cell surface at weekly intervals after BMP has been infused followed by a Noggin infusion.

Note: Initially, BMP levels rise above the initial condition, but the influx of Noggin is able to bring those levels down.

will be affected by perturbations of that parameter value and are collectively defined as

$$
S_{i, j}=\frac{\partial x_{i}}{\partial p_{j}}
$$

where $x_{i}$ refers to each of the state variables and $p_{j}$ refers to each parameter in the model (Dasika, Kinsey, \& Locke, 2012).

We perform a local sensitivity analysis on the non-dimensional base model, focusing on the specific set of nominal parameter values cited above. We are specifically interested in the parameter ranges which reproduce experimental results, and in the relative sensitivities of the parameters. To compute the SC for Noggin, BMP, and the complex with respect to all parameters, we use $S_{i, j}=\frac{\partial x_{i}}{\partial p_{j}}$ and take the time derivative. An example is shown below.

To compute the SC for Noggin with respect to $D_{N C}\left(S_{N, D_{N C}}\right)$, we take the time derivative of $S_{N, D_{N C}}=\frac{\partial N}{\partial D_{N C}}$.

$$
\begin{aligned}
\frac{\partial S_{N, D_{N C}}}{\partial t} & =\frac{\partial}{\partial t} \frac{\partial N}{\partial D_{N C}}=\frac{\partial}{\partial D_{N C}} \frac{\partial N}{\partial t}=\frac{\partial}{\partial D_{N C}}\left(D_{N C} \frac{\partial^{2} N}{\partial x^{2}}-\alpha N B-\beta N\right) \\
& =\frac{\partial^{2} N}{\partial x^{2}}+D_{N C} \frac{\partial}{\partial D_{N C}} \frac{\partial^{2} N}{\partial x^{2}}-\alpha\left(\frac{\partial N}{\partial D_{N C}} B+\frac{\partial B}{\partial D_{N C}} N\right)-\beta \frac{\partial N}{\partial D_{N C}} \\
& =\frac{\partial^{2} N}{\partial x^{2}}+D_{N C} \frac{\partial^{2} S_{N, D_{N C}}}{\partial x^{2}}-\alpha\left(S_{N, D_{N C}} B+S_{B, D_{N C}} N\right)-\beta S_{N, D_{N C}}
\end{aligned}
$$

Since the initial conditions for $N, B$ and $C$ are constant, applying Equation (26) with respect to all parameters to these initial conditions will result in zero. Similarly, applying Equation (26) to all constant boundary conditions with respect to all parameters will result in zero. However, on the right, the flux of BMP is assumed to be dependent on the amount of BMP present at the cell surface. 


$$
\frac{\partial B}{\partial x}(1, t)=m \frac{B(1, t)}{B(1, t)+1}
$$

Therefore, applying Equation (26) to this boundary condition yields

$$
\frac{\partial S_{B, D_{N C}}}{\partial x}(1, t)=m \frac{S_{B, D_{N C}}(1, t)}{(B(1, t)+1)^{2}}
$$

Similarly, we obtain the following non-trivial boundary conditions on the right for the following sensitivity coefficients.

$$
\begin{aligned}
\frac{\partial S_{B, D_{B C}}}{\partial x}(1, t) & =m \frac{S_{B, D_{B C}}(1, t)}{(B(1, t)+1)^{2}} \\
\frac{\partial S_{B, \alpha}}{\partial x}(1, t) & =m \frac{S_{B, \alpha}(1, t)}{(B(1, t)+1)^{2}} \\
\frac{\partial S_{B, \beta}}{\partial x}(1, t) & =m \frac{S_{B, \beta}(1, t)}{(B(1, t)+1)^{2}}
\end{aligned}
$$

The resulting system of equations (the three original non-dimensional reaction-diffusion Equations (13-15) along with the 12 equations for each sensitivity coefficient $S_{i, j}$ in addition to initial and boundary conditions) was solved in Matlab and the steady state concentrations were computed. In the steady-state, the signalling level of BMP at the cell surface is not very sensitive to any parameter, though it is least sensitive to $D_{N C}$. All SCs approach zero. In the beginning of the experiments, the signalling levels are sensitive to $D_{B C}$ and $\beta$. In terms of the dimensional system, this means that the ratio of the degradation rates of free and bound Noggin is important, as is the ratio of the diffusion coefficients of BMP and the complex (Figure 7) (Appendix 1).

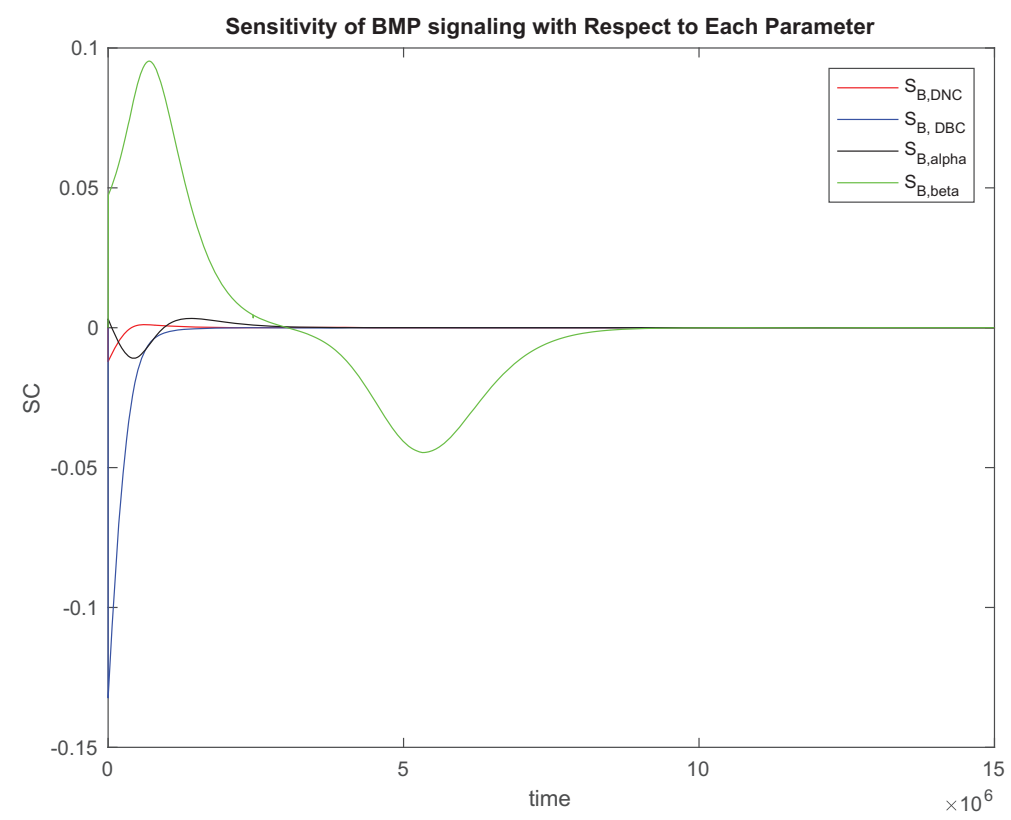

Figure 7. Sensitivity coefficients at the cell surface for BMP with respect to each parameter. 


\subsection{The role of diffusion}

As mentioned above, what is particularly interesting is that BMP-binding proteins can cause context-specific gains or losses in signalling, either by acting as inhibitors and sequestering BMP ligands to prevent them from binding to the cell surface, or by binding and shuttling BMP to receptors. When BMP is bound to a protein such as Noggin or Chordin, the complex is able to diffuse much faster through the extracellular space (Ben-Zvi et al., 2008) which can actually increase signalling. It has been shown that the establishment of a BMP gradient in some cases is really a result of long range inhibition by Noggin and Chordin (Jones and Smith, 1998).

Simulations for different diffusion coefficients show that increasing the diffusion coefficient of Noggin over several orders of magnitude lowers the BMP signalling level at the cell surface. Qualitatively the trend is the same for a variety of tested values. Since a threshold level of BMP signalling is responsible for keeping cells in quiescence, a faster diffusing antagonist could play a pivotal role in bringing cells out of quiescence in response to an influx of Noggin.

If BMP diffuses as rapidly as the complex $\left(D_{B C}=1\right)$ then any infused Noggin has a large and immediate impact on BMP signalling, reducing it to very low levels (see Figure 8(c)). If this were true, there would likely be more of a switch in terms of Noggin affecting quiescence instead of a gradient. This makes our model, though simple, in agreement with what is qualitatively know about the biology and with the experiments discussed throughout.

Turing (1952) showed that a system of proteins undergoing reaction and diffusion can lead to diffusion-driven spatial heterogeneity. That is, a spatially uniform steady state, stable in the absence of diffusion, could be driven unstable by diffusion, evolving into a spatially heterogeneous state. For two species, a key condition for diffusion-driven instability is a difference in magnitude of diffusion coefficients, and rigorous conditions on the linear stability matrix are well known and found in many texts (Murray, 2001). Because our system has an activator (BMP), an inhibitor (Noggin) and a complex, and because experimental evidence suggests their diffusion coefficients are different, we wish to explore the role of diffusion in our system in this context.

In activator-inhibitor models it is common to consider the behaviour of the kinetics without diffusion (Harrington \& Law, 2006; Kicheva et al., 2007). We specifically investigate the role of diffusion in our system by considering our non-dimensionalized base model without diffusive terms. This yields a system of three ordinary differential equations and we consider the equilibria and their stability.

$$
\begin{aligned}
& \frac{\mathrm{d} N}{\mathrm{~d} t}=-\alpha N B-\beta N \\
& \frac{\mathrm{d} B}{\mathrm{~d} t}=-\alpha N B+C \\
& \frac{\mathrm{d} C}{\mathrm{~d} t}=\alpha N B-C
\end{aligned}
$$

In this situation, there are two equilibria. The first equilibrium has a degree of freedom: $N=0, C=0$, and $B$ can be any constant. The second equilibrium: $N=-C / \beta, B=-\beta / \alpha$, $C=-\beta N$ does not make sense biologically, because the proteins cannot have a negative 


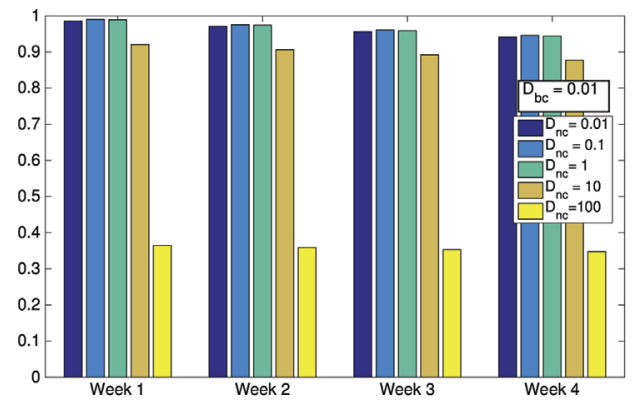

(a) $D_{B C}=.01$ and $D_{N C}$ varies over 5 orders of magnitude.

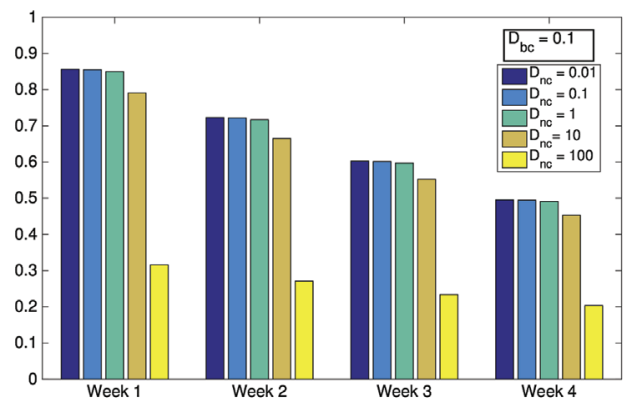

(b) $D_{B C}=.1$ and $D_{N C}$ varies over 5 orders of magnitude.

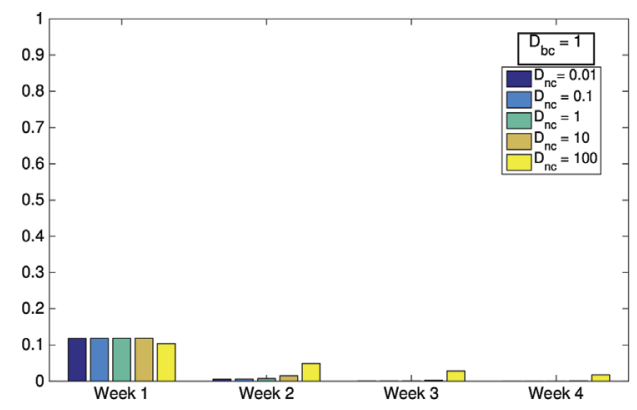

(c) $D_{B C}=1$ and $D_{N C}$ varies over 5 orders of magnitude. This case allows BMP to diffuse at the same speed as the complex. This is not biologically realistic, and the results are not graded.

Figure 8. The BMP signalling level at the cell surface by week as $D_{N C}$ varies for three different values of $D_{B C}$.

Notes: Notice that Figure 8 (c) has significantly reduced BMP signalling compared to the other two cases. When $D_{B C}=.1$ we see that changes in $D_{N C}$ have graded effects on the BMP levels. This supports the parameter choices for the numerical simulation and also supports the biological claim that the complex diffuses faster than BMP.

concentration. Calculating the Jacobian matrix at the first equilibrium yields

$$
\left(\begin{array}{lrr}
-\alpha B-\beta & 0 & 0 \\
-\alpha B & 0 & 1 \\
\alpha B & 0 & -1
\end{array}\right)
$$

The eigenvalues are $\lambda_{1}=-1, \lambda_{2}=0, \lambda_{3}=-\alpha B-\beta$.

We are able to reduce our system by adding Equations (34) to (35). This implies that

$$
B+C=K
$$

where $K$ is a constant. Since $K$ is equal to the sum of $B$ and $C$ at any time, it is specifically equal to the sum of those initial values: $K=B_{0}+C_{0}$.

Solving for $C$ and replacing $C$ in (34), we have the following reduced system.

$$
\frac{\mathrm{d} N}{\mathrm{~d} t}=-\alpha N B-\beta N
$$




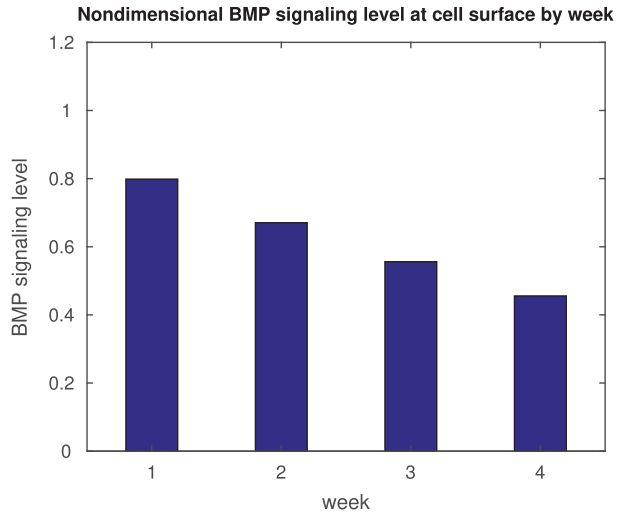

(a) The BMP signaling level at the cell surface by week for the base in vivo model.

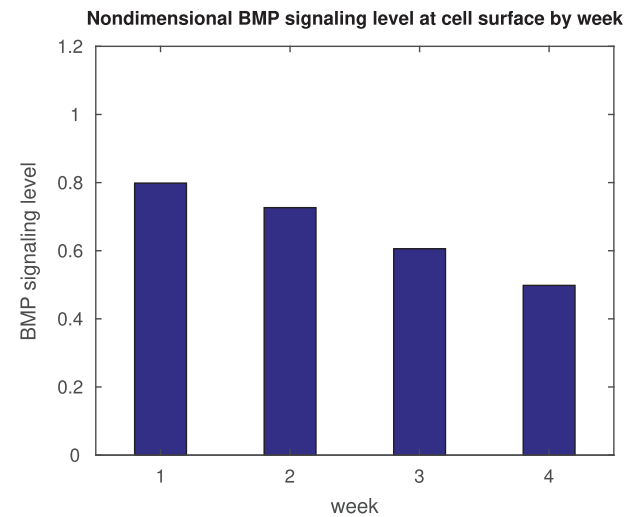

(c) The BMP signaling level at the cell surface by week for the infusion of Noggin experiment done by Mira et al. (2010).

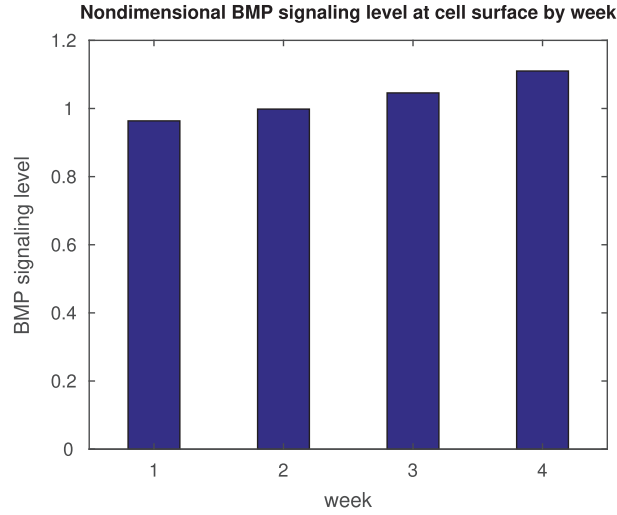

(b) The BMP signaling level at the cell surface by week for the base in vivo model with autocrine signaling.

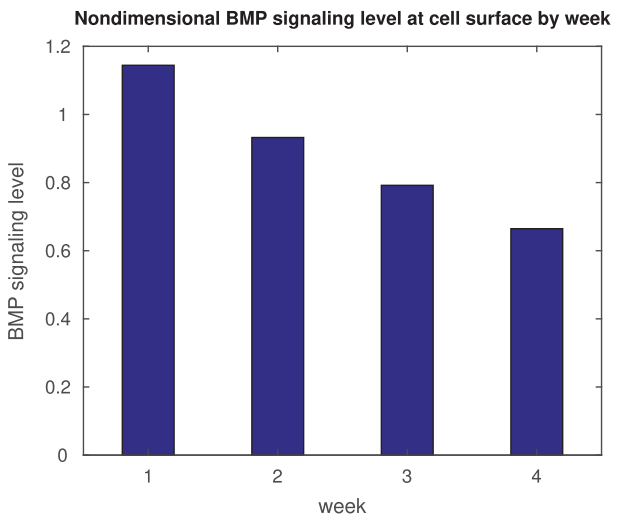

(d) The BMP signaling level at the cell surface by week for the theoretical infusion of BMP experiment suggested by Mira et al. (2010)

Figure 9. A comparison of the BMP signalling profile for the in vivo model, the in vivo model with autocrine signalling, the infusion of Noggin experiment, and the theoretical infusion of BMP experiment.

$$
\frac{\mathrm{d} B}{\mathrm{~d} t}=-\alpha N B-B+K
$$

This system has a unique equilibrium at $(0, K)$ and we can use phase plane analysis to show it is a stable node. The Jacobian has eigenvalues $\lambda_{1}=-1, \lambda_{2}=-\alpha K-\beta$. Both are negative.

In the three-dimensional system, this means that in the absence of diffusion, all trajectories approach $(0, K, 0)$ where $K$ is determined by initial conditions. Noggin degrades, and without a boundary influx or diffusion, its concentration goes to zero. The time derivative of Noggin is negative for all time, so its concentration decreases. If Noggin goes to zero, 
the differential equation governing the complex is

$$
\frac{\mathrm{d} C}{\mathrm{~d} t} \approx-C
$$

so the complex concentration always decreases. The equation governing BMP is

$$
\frac{\mathrm{d} B}{\mathrm{~d} t} \approx 0
$$

so BMP goes to a constant value.

This analysis shows that diffusion is necessary to have any meaningful signalling profiles and in its absence, the system equilibrates to a constant level of BMP that depends specifically on the initial conditions.

Qian and Murray (2001) derive a simple and practical rule for reaction-diffusion equations of three species to have diffusion-driven instability. They find that a necessary and sufficient condition for diffusion-driven instability with three species is that either the largest diagonal element of $A$ is greater than zero or the smallest diagonal cofactor of $A$ is less than zero where $A$ is the Jacobian matrix from linearization about the steady state.

For our matrix $A$, all diagonal elements are less than or equal to zero and there is only one diagonal non-zero cofactor which is positive.

$$
C_{2,2}=(-1)^{4}(\alpha B+\beta)
$$

Thus, it is not possible to have diffusion-driven instability in this system.

The condition above can be thought of as a generalization of the condition with two species. For two species, to have diffusion-driven instability, the diffusion coefficient of the inhibitor must be larger than that of the activator. The generalization for three species is that there are either two inhibitors and one activator, or two activators and one inhibitor (Qian \& Murray, 2001). In our system, this is not a surprise, because the complex is not able to either activate NSCs through binding or inhibit BMP.

\section{Discussion and conclusions}

In this paper, we have presented a mathematical model for the densities of BMP and Noggin in the adult hippocampus. We have considered how the balance of these molecules creates signalling profiles in different situations. This model has generated the theoretical BMP signalling profiles that relate to the experiments conducted by Mira et al. (2010) (particularly in Figure 6), and has hypothetically demonstrated that transient BMP infusion leads to a profile that can be reversed by infusion of Noggin (Figure 9).

Additionally, we have explored the possible autocrine nature of BMP regulation with our model. Mira et al. (2010) suggest that NSCs may regulate proliferation in an autocrine manner by secreting BMP. Bonaguidi et al. (2008) note that neural precursors also secrete BMP, potentially affecting signalling in a paracrine manner. By modelling the autocrine and paracrine signalling through the production of BMP in our reaction diffusion model, the signalling profile grows each week. This suggests that if autocrine signalling is occurring, there is an additional mechanism which dampens the signalling level, or that the NSCs become attenuated to the level of BMP created. Yousef et al. (2015) find that attenuation 
of BMP signalling enhances neurogenesis. How this attenuation occurs is important to elucidate. Ideally, future experiments will focus on the possible autocrine nature of this signalling mechanism.

We have also considered the role of diffusion in this system by eliminating it from the equations and considering the equilibria. These results show that diffusion is a critical process in this niche, because without it, the necessary signalling profiles will not be achieved. Moreover, we have varied diffusion coefficients over five orders of magnitude and found that faster Noggin diffusion lowers the BMP signalling level at the cell surface as long as $D_{B}<<D_{C}$. A faster diffusing antagonist could play a pivotal role in bringing cells out of quiescence rapidly. Moreover, we have shown our system cannot exhibit diffusiondriven instability despite the difference in diffusion coefficients.

The role of BMP in the model is multifaceted. First and foremost, it is a signal to the cell to remain quiescent. By also considering the autocrine nature of its signalling, it has a role in the development of the signalling profile, effectively amplifying the signal. Lastly, by binding to Noggin and diffusing more rapidly in the bound form, it has a counterintuitive role with its antagonist.

It should be pointed out that the model presented here is not complete and there are a number of assumptions that we have made about the system, such as the existence of a protease for Noggin analogous to Xolloid for Chordin or to Tolloid for Sog. There are other signalling molecules that interact with both Noggin and BMP, and those are excluded from the model. For example, another regulatory mechanism by which the activity of BMP signalling proteins is modulated involves ubiquitin-mediated proteasomal degradation (Cao \& Chen, 2005). An extension of our model could include a signalling cascade of ubiquitin transfer reactions through the three required enzymes. Additionally, Smad6 plays a negative role in BMP signalling by binding to BMP receptors. The expression of Smad6 is regulated by BMPs (Cao \& Chen, 2005; Mira et al., 2010). This could be an interesting dynamic to explore with a future model. We also group several BMPs in our model, and although they can often compensate for each other (Tsuji et al., 2008) the individual BMPs are not ubiquitous and have slight differences in function (e.g. BMP2/4 vs. BMP5).

Another limitation of our model is that we consider a highly simplified geometry. A natural future project would be to consider a two or three dimensional geometry. Mira et al. (2010) includes in vitro experiments, and it is well known that the effects of Noggin are density-dependent (Bonaguidi et al., 2008) (i.e. the neural stem cells may start producing Noggin themselves under certain conditions). In fact, one of those conditions is stimulation by BMP4 itself (Feeley et al., 2006). In general, regulation of neural precursor cells is cell density-dependent (Limoli et al., 2004), and density is neglected in our onedimensional model.

Our work relies on results from two studies in which Noggin was administered to hippocampal NSCs (Bonaguidi et al., 2008; Mira et al., 2010). Recombinant Noggin, as administered in these papers, does not always show the same biological effect as endogenous Noggin, which complicates the conclusions from studies with overexpression of recombinant protein, for example by pumps, compared to when endogenous Noggin signalling is blocked. 
However, despite the simplicity of our model, it is a useful tool to mimic the qualitative dynamics of biological experiments and to predict that the transient infusion of BMP leads to a signalling profile that is indeed reversible by an infusion of Noggin.

Mira et al. (2010) note that Noggin infusion by pump initially increases cell proliferation, but by three weeks, the active cells have decreased. This result is different than Bonaguidi et al. (2008), and the authors suggest it could be from the acute delivery of Noggin from the pump rather than from a continuous exposure. Our equations model the pump infusion continuously (just as Noggin comes from the vasculature) and our results agree more closely with Bonaguidi et al. (2008). We conclude that continuous Noggin exposure will result in more actively dividing cells. Our model links the Noggin exposure to the decreasing BMP signalling profile, which allows cells to emerge from quiescence and to actively divide.

It has recently been shown that hippocampal BMP signalling is modulated by antidepressant treatment, in that antidepressants suppress BMP signalling and increase the production of Noggin (Brooker, Gobeske, Chen, Peng, \& Kessler, 2016). This makes BMP signalling a powerful potential target for the treatment of depression. Physical exercise is tied to Noggin production (Gobeske et al., 2009) and also to better outcomes in depression (Stanton \& Reaburn, 2014). The balance of BMP and Noggin links these two relationships.

BMP signalling has also recently been shown to increase with age and contribute to an age-related decline in cognitive function (Yousef et al., 2015). Moreover, the authors found that the genetic inhibition of BMP signalling partially rescued neurogenesis. This ability to rescue cell function by modulating signal levels in the NSC hippocampal niche suggests promising strategies for addressing the loss of cognitive function over time. This study did not address the infusion of Noggin, but that would be an important future experiment. It would also be interesting to test whether there is more autocrine signalling as an organism ages, as our model shows autocrine signalling keeps BMP levels high.

BMP signalling regulates NSC number, proliferation, and cell fate commitment. We have uses our reaction-diffusion model to investigate the balance of BMP and Noggin and the role of diffusion in the signalling profiles in the adult hippocampal stem cell niche.

\section{Acknowledgements}

The authors wish to thank Alex Mogilner for providing valuable feedback in the early stages of the project, Chris Dugaw for discussions about stability, and Aaron Tempelaere for assistance with figures.

\section{Disclosure statement}

No potential conflict of interest was reported by the authors.

\section{References}

Badugu, A., Kraemer, C., Germann, P., Menshykau, D., \& Iber, D. (2013). Digit patterning during limb development as a result of the BMP-receptor interaction. arXiv preprint arXiv:1301.0736.

Balemans, W., \& Van Hul, W. (2002). Extracellular regulation of BMP signaling in vertebrates: A cocktail of modulators. Developmental Biology, 250(2), 231-250.

Ben-Zvi, D., Shilo, B.-Z., Fainsod, A., \& Barkai, N. (2008). Scaling of the BMP activation gradient in Xenopus embryos. Nature, 453(7199), 1205-1211. 
Bonaguidi, M. A., Peng, C.-Y., McGuire, T., Falciglia, G., Gobeske, K. T., Czeisler, C., \& Kessler, J. A. (2008). Noggin expands neural stem cells in the adult hippocampus. Journal of Neuroscience, 28(37), 9194-9204.

Brooker, S. M., Gobeske, K. T., Chen, J., Peng, C.-Y., \& Kessler, J. A. (2016). Hippocampal bone morphogenetic protein signaling mediates behavioral effects of antidepressant treatment. Molecular Psychiatry, 22, 910-919.

Cao, X., \& Chen, D. (2005). The BMP signaling and in vivo bone formation. Gene, 357(1), 1-8.

Chapouton, P., Skupien, P., Hesl, B., Coolen, M., Moore, J. C., Madelaine, R., ... Bally-Cuif, L. (2010). Notch activity levels control the balance between quiescence and recruitment of adult neural stem cells. Journal of Neuroscience, 30(23), 7961-7974.

Coller, H. A. (2011). The essence of quiescence. Science, 334(6059), 1074-1075.

Dasika, S. K., Kinsey, Stephen T., \& Locke, B. R. (2012). Sensitivity analysis of reaction-diffusion constraints in muscle energetics. Biotechnology and Bioengineering, 109(2), 559-571.

De Robertis, E. M., Wessely, O., Oelgeschläger, M., Brizuela, B., Pera, E., Larraín, J., \& Abreu, J. (2001). Molecular mechanisms of cell-cell signaling by the Spemann-Mangold organizer. The International Journal of Developmental Biology, 45(1), 189.

Eldar, A., Dorfman, R., Weiss, D., Ashe, H., et al. (2002). Robustness of the BMP morphogen gradient in Drosophila embryonic patterning. Nature, 419(6904), 304-308.

Eroshkin, F. M., Nesterenko, A. M., Borodulin, A. V., Martynova, N. Y., Ermakova, G. V., Gyoeva, F. K., ... Zaraisky, A. G. (2016). Noggin4 is a long-range inhibitor of Wnt8 signalling that regulates head development in Xenopus laevis. Scientific Reports, 6. Retrieved from https://www. researchgate.net/profile/Andrey_Zaraisky/publication/298338684_Noggin4_is_a_long-range_ inhibitor_of_Wnt8_signalling_that_regulates_head_development_in_Xenopus_laevis/links/ 56e8107a08aec65cb45e82e3.pdf

Feeley, B. T., Krenek, L., Liu, N., Hsu, W. K., Gamradt, S. C., Schwarz, E. M., ... Lieberman, J. R. (2006). Overexpression of noggin inhibits BMP-mediated growth of osteolytic prostate cancer lesions. Bone, 38(2), 154-166.

Francois, P., Vonica, A., Brivanlou, A. H., \& Siggia, E. D. (2009). Scaling of BMP gradients in Xenopus embryos. Nature, 461(7260), E1-E1.

Freeman, M. (2000). Feedback control of intercellular signalling in development. Nature, 408(6810), 313-319.

Gobeske, K. T., Das, S., Bonaguidi, M. A., Weiss, C., Radulovic, J., Disterhoft, J. F., \& Kessler, J. A. (2009). BMP signaling mediates effects of exercise on hippocampal neurogenesis and cognition in mice. PloS One, 4(10), e7506.

Hama, H., Kurokawa, H., Kawano, H., Ando, R., Shimogori, T., Noda, H., ... Miyawaki, A. (2011). Scale: A chemical approach for fluorescence imaging and reconstruction of transparent mouse brain. Nature Neuroscience, 14(11), 1481.

Harrington, H. A., \& Law, K. J. H. (2006). Creating patterns reaction-diffusion theory. Retrieved from http://infohost.nmt.edu/ starrett/Spring2015/530/ReactionDiffusion/PatternsFinalPaper.pdf

Ingalls, B. (2008). Sensitivity analysis: From model parameters to system behaviour. Essays in Biochemistry, 45, 177-194.

Inomata, H., Shibata, T., Haraguchi, T., \& Sasai, Y. (2013). Scaling of dorsal-ventral patterning by embryo size-dependent degradation of Spemann's organizer signals. Cell, 153(6), 1296-1311.

Johnston, M. A., \& Lim, D. A. (2010). Keeping them quiet: BMPs maintain adult neural stem cell quiescence. Cell Stem Cell, 7(1), 9-10.

Jones, C. M., \& Smith, J. C. (1998). Establishment of a BMP-4 morphogen gradient by long-range inhibition. Developmental Biology, 194(1), 12-17.

Karim, M. S., Buzzard, G. T., \& Umulis, D. M. (2012). Secreted, receptor-associated bone morphogenetic protein regulators reduce stochastic noise intrinsic to many extracellular morphogen distributions. Journal of The Royal Society Interface, 9(70), 1073-1083.

Kawaguchi, D., Furutachi, S., Kawai, H., Hozumi, K., \& Gotoh, Y. (2013). Dll1 maintains quiescence of adult neural stem cells and segregates asymmetrically during mitosis. Nature Communications, 4,1880 . 
Kicheva, A., Pantazis, P., Bollenbach, T., Kalaidzidis, Y., Bittig, T., Jülicher, F., \& González-Gaitán, M. (2007). Kinetics of morphogen gradient formation. Science, 315(5811), 521-525.

Lander, A. D., Kimble, J., Clevers, H., Fuchs, E., Montarras, D., Buckingham, M., ... Oskarsson, T. (2012). What does the concept of the stem cell niche really mean today? BMC Biology, 10(1), 19.

Lim, D. A., Tramontin, A. D., Trevejo, J. M., Herrera, D. G., García-Verdugo, J. M., \& Alvarez-Buylla, A. (2000). Noggin antagonizes BMP signaling to create a niche for adult neurogenesis. Neuron, 28(3), 713-726.

Limoli, C. L., Rola, R., Giedzinski, E., Mantha, S., Huang, T.-T., \& Fike, J. R. (2004). Cell-densitydependent regulation of neural precursor cell function. Proceedings of the National Academy of Sciences of the United States of America, 101(45), 16052-16057.

Marqués, G., Musacchio, M., Shimell, M. J., Wünnenberg-Stapleton, K., Cho, K. W. Y., \& O’Connor, M. B. (1997). Production of a DPP activity gradient in the early Drosophila embryo through the opposing actions of the SOG and TLD proteins. Cell, 91(3), 417-426.

Mira, H., Andreu, Z., Suh, H., Lie, D. C., Jessberger, S., Consiglio, A., ... Gage, F. H. (2010). Signaling through BMPR-IA regulates quiescence and long-term activity of neural stem cells in the adult hippocampus. Cell Stem Cell, 7(1), 78-89. Retrieved from https://ac.els-cdn.com/S1934590910001712/ 1-s2.0-S1934590910001712-main.pdf?_tid=57b57ad6-c6fb-11e7-8c82-00000aacb35f\& acdnat $=1510417026 \_7464 \mathrm{c} 93134 \mathrm{~b} 254 \mathrm{~d} 1 \mathrm{f} 228789 \mathrm{f} 266 \mathrm{a} 8500$

Mizutani, C. M., Nie, Q., Wan, F. Y. M., Zhang, Y.-T., Vilmos, P., Sousa-Neves, R., ... Lander, A. D. (2005). Formation of the BMP activity gradient in the Drosophila embryo. Developmental Cell, $8(6), 915-924$.

Murray, J. D. (2001). Mathematical biology. II spatial models and biomedical applications \{Interdisciplinary Applied Mathematics V. 18\}. New York: Springer-Verlag New York Incorporated.

Nerurkar, N. L., Mahadevan, L., \& Tabin, C. J. (2017). BMP signaling controls buckling forces to modulate looping morphogenesis of the gut. Proceedings of the National Academy of Sciences, 114(9), 2277-2282.

O'Connor, M. B., Umulis, D., Othmer, H. G., \& Blair, S. S. (2006). Shaping BMP morphogen gradients in the Drosophila embryo and pupal wing. Development, 133(2), 183-193.

Oelgeschläger, M., Larraín, J., Geissert, D., \& De Robertis, E. M. (2000). The evolutionarily conserved BMP-binding protein twisted gastrulation promotes BMP signalling. Nature, 405(6788), 757-763.

Percus, J. K., \& Childress, S. (2015). Mathematical models in developmental biology, Vol. 26. American Mathematical Society.

Piccolo, S., Agius, E., Lu, B., Goodman, S., Dale, L., \& De Robertis, E. M. (1997). Cleavage of Chordin by Xolloid metalloprotease suggests a role for proteolytic processing in the regulation of Spemann organizer activity. Cell, 91(3), 407-416.

Qian, H., \& Murray, J. D. (2001). A simple method of parameter space determination for diffusiondriven instability with three species. Applied Mathematics Letters, 14(4), 405-411.

Rentzsch, F., Zhang, J., Kramer, C., Sebald, W., \& Hammerschmidt, M. (2006). Crossveinless 2 is an essential positive feedback regulator of Bmp signaling during zebrafish gastrulation. Development, 133(5), 801-811.

Snyder, Evan Y. (2011). The risk of putting something where it does not belong: Mesenchymal stem cells produce masses in the brain. Experimental Neurology, 230(1), 75-77.

Spill, F., Guerrero, P., Alarcon, T., Maini, P. K., \& Byrne, H. M. (2015). Mesoscopic and continuum modelling of angiogenesis. Journal of Mathematical Biology, 70(3), 485-532.

Stanton, R., \& Reaburn, P. (2014). Exercise and the treatment of depression: A review of the exercise program variables. Journal of Science and Medicine in Sport, 17(2), 177-182.

Stantzou, A., Schirwis, E., Swist, S., Alonso-Martin, S., Polydorou, I., Zarrouki, F., ... Helge, A. (2017). BMP signaling regulates satellite cell dependent postnatal muscle growth. Development, dev-144089.

Syková, E., \& Nicholson, C. (2008). Diffusion in brain extracellular space. Physiological Reviews, 88(4), 1277-1340. 
Tsuji, K., Cox, K., Bandyopadhyay, A., Harfe, B. D., Tabin, C. J., \& Rosen, V. (2008). BMP4 is dispensable for skeletogenesis and fracture-healing in the limb. JBJS, 90 (Supplement_1), 14-18.

Turing. (1952). The chemical basis of morphogenesis. Sciences-cecm. usp. br.

Umulis, D., O'Connor, M. B., \& Blair, S. S. (2009). The extracellular regulation of bone morphogenetic protein signaling. Development, 136(22), 3715-3728.

Umulis, D. M., Serpe, M., O’Connor, M. B., \& Othmer, H. G. (2006). Robust, bistable patterning of the dorsal surface of the Drosophila embryo. Proceedings of the National Academy of Sciences, 103(31), 11613-11618.

Yousef, H., Morgenthaler, A., Schlesinger, C., Bugaj, L., Conboy, I. M., \& Schaffer, D. V. (2015). Age-associated increase in BMP signaling inhibits hippocampal neurogenesis. Stem Cells, 33(5), 1577-1588.

Yu, S. R., Burkhardt, M., Nowak, M., Ries, J., Petrásek, Z., Scholpp, S., ... Brand, M. (2009). Fgf8 morphogen gradient forms by a source-sink mechanism with freely diffusing molecules. Nature, 461(7263), 533.

Zaraisky, A. G. (2007). Neural induction: New achievements and prospects. Molecular Biology, 41(2), 173-186.

Zhou, S., Lo, W.-C., Suhalim, J. L., Digman, M. A., Gratton, E., Nie, Q., \& Lander, A. D. (2012). Free extracellular diffusion creates the Dpp morphogen gradien0t of the Drosophila wing disc. Current Biology, 22(8), 668-675.

\section{Appendix 1. Steady state analysis}

We are interested in the limiting case of the more general model and in the BMP, Noggin and complex profile as measured in the steady state. We assume that BMP diffuses significantly more slowly than Noggin or the complex, so we let $D_{B C}=0$. This assumption coupled with forcing all time derivatives to be zero in the steady-state yields the following equations.

$$
\begin{aligned}
0 & =D_{N C} \frac{\partial^{2} N}{\partial x^{2}}-\alpha N B-\beta N \\
0 & =-\alpha N B+C \\
0 & =\frac{\partial^{2} C}{\partial x^{2}}+\alpha N B-C
\end{aligned}
$$

By adding Equations (A2) to (A3) we find

$$
0=\frac{\partial^{2} C}{\partial x^{2}}
$$

Solving this equations with zero flux boundary conditions at $x=0$, we find that

$$
C=C_{0} .
$$

By adding Equations (A1) and (A3) and substituting our result, we have

$$
0=D_{N C} \frac{\partial^{2} N}{\partial x^{2}}-\beta N-C_{0}
$$

This is a non-homogenous second-order ordinary differential equation, so we use the principle of superposition to obtain a general solution, which is

$$
N(x)=N_{h}+N_{p}=c_{1} e^{\sqrt{\frac{\beta}{D_{N C}}} x}+c_{2} e^{-\sqrt{\frac{\beta}{D_{N C}}} x}-\frac{C_{0}}{\beta} .
$$

We can then use the given boundary conditions to solve for the constants. 
Qualitatively, this function shows that Noggin rises in density as the space variable increases. Now that we have expressions for $N$ and $C$, we use Equation (A3), make appropriate substitutions and solve algebraically for $B$.

$$
B=\frac{C_{0}-\alpha}{c_{1} e^{\sqrt{\frac{\beta}{D_{N C}}} x}+c_{2} e^{-\sqrt{\frac{\beta}{D_{N C}}} x}-\frac{C_{0}}{\beta}}
$$

We leave $c_{1}$ and $c_{2}$ in this equation for simplicity. 